\title{
Measurement of Mouse Heart Rate Variability using Echocardiographic System
}

\author{
Filipe Fernandes Stoyell-Conti ${ }^{1,2}$, Fernando Santos ${ }^{2,3}$, Jacqueline Freire Machi $23,3,4$, Diana Rosa Hernandez ${ }^{2,4}$, Catarina Andrade Barboza ${ }^{2}$, \\ Maria-Cláudia Irigoyen ${ }^{3}$, Kátia De Angelis ${ }^{1,3,5 *}$, Mariana Morris ${ }^{2,4 *}$ \\ 1 Laboratory of Translational Physiology, Nove de Julho University, ${ }^{3}$ Hypertension Unit, Heart Institute (InCor), School of Medicine, University of Sao Paulo, \\ ${ }^{5}$ Department of Physiology, Federal University of Sao Paulo, Sao Paulo, Brazil, ${ }^{2}$ Institute of Neuro-Immune Medicine, Nova Southeastern University, \\ ${ }^{4}$ Miami VA Healthcare System, FL, USA \\ *These authors equally contributed as senior authors
}

\section{Abstract}

\begin{abstract}
Aim: We employed an echocardiographic (ECHO) system as the backbone for the collection of electrocardiogram (ECG) and heart rate variability (HRV) data. The system was tested using an exercise model in which C57 male mice were exposed to sham or forced wheel running. Methods: Peak/peak (RR) interval was recorded over a 3 min period using the ECG platform of the ECHO system. Isoflurane-anesthetized male mice were divided into two groups ( $n=8$ /group): sedentary (S) and forced wheel trained (T). HRV was analyzed in time and frequency domains (Fast Fourier Transform). Exercise training (T) was performed on a motorized wheel at low intensity 1 h/day, 5 days/week, 8 weeks duration. Cardiac morphometry and function were analyzed using ECHO while ECG was the basis to measure HRV. The sampling rate was $8000 \mathrm{~Hz}$. Results show that the trained mice presented a reduction in heart rate as compared to the sedentary group. This was associated with lower cardiac sympathetic and higher parasympathetic modulation leading to an improved sympathetic/parasympathetic ratio (low-frequency band/high-frequency band). The trained group showed a reduction in isovolumetric relaxation time, reduced myocardial performance index, increased relative wall thickness, and left ventricle mass when compared to the sedentary group. Conclusion: Results document the utility of combining the ECHO and the ECG platform, allowing for the dual measurement of autonomic and cardiac function in mice.
\end{abstract}

Keywords: Echocardiography, electrocardiogram, exercises training, heart rate variability, mice, noninvasive method

\section{INTRODUCTION}

Murine animal species have been utilized to gain insight into cardiovascular function, especially autonomic control. For the current study, our group will validate the use of an electrocardiogram (ECG) platform coupled with echocardiogram (ECHO) equipment for the acquisition of R-to-R interval (RRI) data for spectral analysis of heart rate variability (HRV) using a murine animal model.

HRV is widely used as a tool to assess the role of autonomic nervous system fluctuations in normal healthy individuals and in patients with various cardiovascular and noncardiovascular disorders. ${ }^{[1]}$ It is important to emphasize that reduction in $\mathrm{HRV}$ is associated with negative cardiovascular outcomes..$^{[2,3]}$ This is seen with hypertension, stroke, diabetes, and other chronic diseases. ${ }^{[3,4]}$ The question is directed toward the issue whether there can be dual recordings of cardiac function and

\begin{tabular}{|l|l|}
\hline \multicolumn{3}{|c|}{ Access this article online } \\
\hline Quick Response Code: & Website: \\
& www.jcecho.org \\
& \\
&
\end{tabular}

ECG using a unique system. While ECHO is commonly used to provide cardiac function and morphometric, it has been largely overlooked the use of its ECG platform (except for monitoring heart rate [HR] and anesthesia status). Indeed, it is possible using this platform, to record the peak/peak RRI, which is used to analyze cardiac autonomic modulation using spectral analysis of HRV at the same time of cardiac function and morphometric measurements.

The quality of the signal, RRI from the ECG or pulse interval derivate from direct BP recording, is a crucial component for

Address for correspondence: Dr. Mariana Morris, Nova Southeastern University, Institute Neuro Immune Medicine 3440 S. University Dr, Fort Lauderdale, Florida 33328, USA E-mail: mmorris1@nova.edu

This is an open access journal, and articles are distributed under the terms of the Creative Commons Attribution-NonCommercial-ShareAlike 4.0 License, which allows others to remix, tweak, and build upon the work non-commercially, as long as appropriate credit is given and the new creations are licensed under the identical terms.

For reprints contact: reprints@medknow.com

How to cite this article: Stoyell-Conti FF, Santos F, Machi JF, Hernandez DR, Barboza CD, Irigoyen MC, et al. Measurement of mouse heart rate variability using echocardiographic system. J Cardiovasc Echography 2018;28:90-4. 


\section{Stoyell-Conti, et al.: Echocardiography for measure of cardiac autonomic function in mice}

HRV analysis. In this aspect, the acquisition of biological signals in mice, particularly cardiovascular signals, is a challenge. This is due to the small animal size, which requires surgical skill as well as specialized, expensive recording equipment. For this reason, the use of ECG platform associated with the ECHO evaluation may represent an alternative methodology to obtain RRI in mice, allowing for analysis of cardiac autonomic modulation.

The aim of this study was to validate the use of the ECG platform coupled with ECHO equipment for the acquisition of RRI data for spectral analysis of HRV in rodents. To test the system for physiological responsiveness, we used chronic forced aerobic exercise training in mice in 8 weeks. Studies from our group have shown that aerobic exercise training promotes resting bradycardia, reduces sympathetic modulation, increases vagal modulation, improves baroreflex responses, and overall cardiac function in models of cardiovascular disease..$^{[5-8]}$

\section{Methods}

\section{Experimental model}

Male mice (C57BI/6J), 8-week-old, were purchased from Jackson Laboratory (ME, USA). Mice were randomly assigned as follows: sedentary $(\mathrm{S} ; n=8)$ and exercise trained $(\mathrm{ET} ; n=8)$. All procedures and protocols were approved by the Ethics Committee of the VA Hospital (Miami, FL, USA) and were conducted in accordance with National Institutes of Health-Guide for the Care and Use of Laboratory Animals.

\section{Exercise training}

Exercise training was performed using a motorized wheel at low intensity $(20 \%$ of the maximal running speed as usually reached in mice) for $1 \mathrm{~h} /$ day, 5 days/week, 8 weeks duration. The speed during the training period was $2 \mathrm{~m} / \mathrm{min}$ and gradually increased to $5 \mathrm{~m} / \mathrm{min}$. Mice were adapted to the procedure $(10 \mathrm{~min} /$ day; $0.2 \mathrm{~m} / \mathrm{min})$ for 1 week before beginning the exercise training protocol. ${ }^{[9]}$

\section{ECG acquisition and heart rate variability analysis}

After the last exercise training session (8 weeks), ECG was recorded at $8000 \mathrm{~Hz}$ for 3 min using a Vevo $1100 \AA$ ultrasound machine (Visual Sonic, Toronto, Canada). Mice were anesthetized with isoflurane (ISO), $\left(2 \%-2.5 \%\right.$ with $\mathrm{O}_{2}$ flow rate of $0.81 / \mathrm{min}$ ). ISO is an excellent mode of anesthesia to use with the (ECO) echocardiogram system. It is stable, rapidly induced and has no long-term side effects. The time required for the ECG measurements is minimal ( $<5 \mathrm{~min}$.), thus, leading to a short window of time needed for anesthesia. After anesthesia induction, mice were secured to a board containing the ECG sensors. ECG was recorded in the ECO B-mode at a frame rate of 5 (minimal value), set using the "Acquisition" key. The cine time loop size was set with the "Prefs" key while the "General" key was set at maximum level for the B-mode. ECG data were exported as a comma-separated value (csv) file and opened with Windaq ${ }^{\circledR}$ Software (DATAQ Instruments). We used an algorithm to detect $\mathrm{R}$ peaks and create a time series file containing all RRIs. Spectral power for low-frequency (low-frequency band [LF]: 0.1-1.0 Hz) and high-frequency (high-frequency band [HF]: 1.0-4.0 Hz) bands were calculated by power spectrum density integration within each frequency bandwidth, using a customized routine (MATLAB 6.0, Mathworks). ${ }^{[1]}$

\section{Echocardiography}

For functional and morphometric analysis, mice were anesthetized with isoflurane as described previously. ECO was performed by an observer blinded as to group, according to the guidelines of the American Society of ECHO. ECO images were obtained with a $10-14 \mathrm{MHz}$ linear transducer with the Vevo 1100® (ultrasound system, Visual Sonic, Toronto, Canada). Measures include left ventricular internal diameter in systole (end-systolic diameter); left ventricular internal diameter in diastole (end-diastolic diameter); ejection fraction (EF); fraction shortening (FS); relative wall thickness (RWT) and isovolumetric relaxation time (IVRT); myocardial performance index (MPI) as previously described elsewhere. ${ }^{[10]}$

\section{Statistical analysis}

Data are expressed as mean \pm standard error mean. The Kolmogorov-Smirnov test was used to evaluate data homogeneity. A $t$-test was used to compare groups. The statistical significance level was established at $P \leq 0.05$.

\section{RESULTS}

ECG and autonomic function data were collected in anesthetized mice under sedentary and exercise conditions. The sample rate for the ECG platform was $8000 \mathrm{~Hz}$, which resulted in a clear interpretable signal [Figure 1].

After 8 weeks of exercise training, the trained group that underwent exercise conditions presented a significant reduction in HR as compared to sedentary mice (S: $453 \pm 21$ vs. T: $387 \pm 18 \mathrm{bpm}$ ) [Figure 2]. This was also associated with lower sympathetic and higher parasympathetic modulation, which was associated with a lower sympathetic/parasympathetic ratio (LF/HF) [Table 1]. An example of the autonomic power spectrum in mice is showed in [Figure 3]. It is possible to see a higher vagal modulation in the trained group when compared to the sedentary group

The trained group that underwent exercise conditions also presented a reduction in IVRT and a better MPI when compared to the sedentary group [Table 2 and Figure 4]. In addition, the trained group showed an increased RWT and left ventricle mass [Table 2]. There was no difference in left ventricular internal diameter in systole, left ventricular internal diameter in diastole, EF, FS, and E/A relation [Table 2].

\section{Discussion}

HRV has been used routinely to evaluate the role of the autonomic nervous and physiological/pathological function. ${ }^{[1]}$ A reduced HRV is associated with negative outcomes, as a 


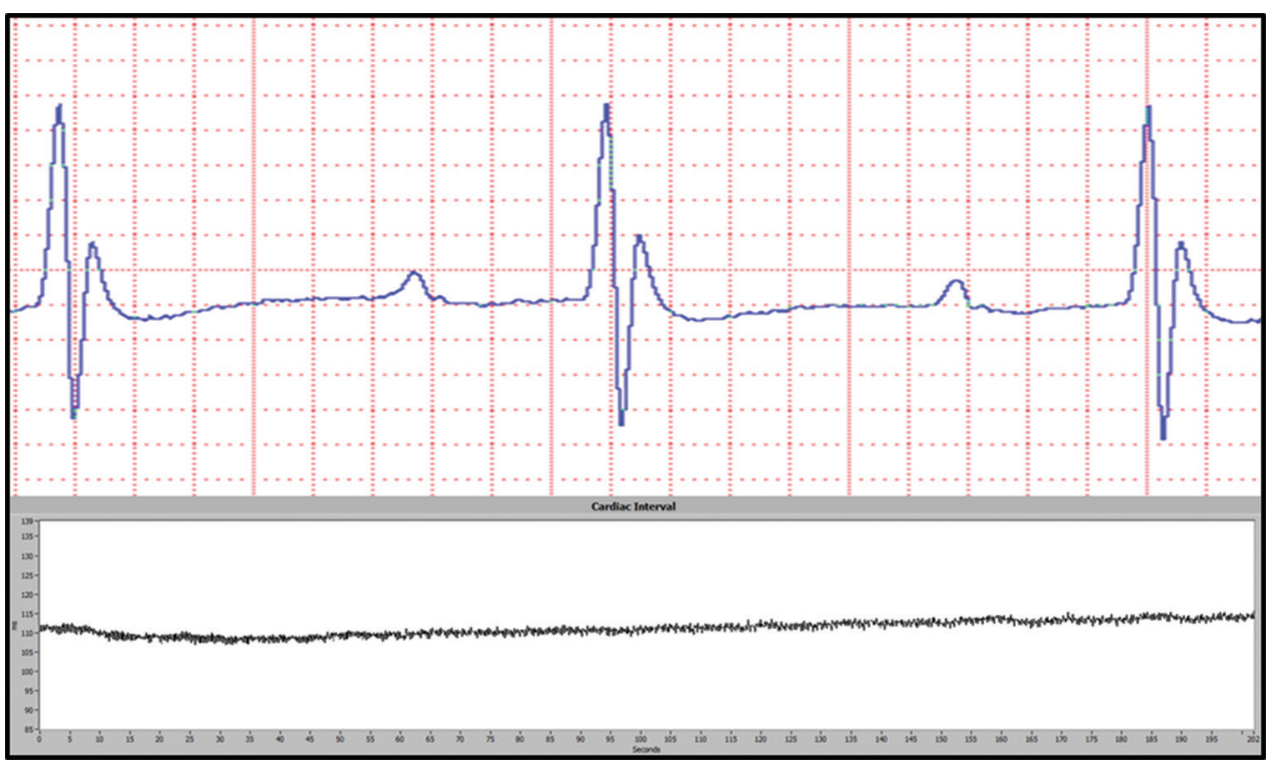

Figure 1: ECG waves signal (superior panel) and tachogram obtained by cardiac interval recording (inferior panel)

\begin{tabular}{lcc}
\hline $\begin{array}{l}\text { Table 1: Autonomic modulation evaluations in studied } \\
\text { groups }\end{array}$ & Trained \\
\hline \multicolumn{2}{l}{ Sedentary } \\
\hline Time domain & & \\
SD (ms) & $4.1 \pm 0.8$ & $5.9 \pm 0.5$ \\
Variance (ms $\left.{ }^{2}\right)$ & $24.7 \pm 9.3$ & $37.2 \pm 7.3$ \\
RMSSD (ms) & $2.8 \pm 0.4$ & $3.1 \pm 0.6$ \\
Frequency domain & & \\
LF abs (ms $\left.{ }^{2}\right)$ & $4.5 \pm 1.7$ & $0.9 \pm 0.2$ \\
HF abs $\left(\mathrm{ms}^{2}\right)$ & $4.5 \pm 0.9$ & $3.7 \pm 1.1$ \\
VLF abs (ms $\left.{ }^{2}\right)$ & $7.5 \pm 3.2$ & $7.3 \pm 1.7$ \\
LF (nu) & $39.2 \pm 6.5$ & $20.7 \pm 5.2^{*}$ \\
HF (nu) & $60.7 \pm 6.5$ & $79.3 \pm 5.2^{*}$ \\
LF/HF & $0.8 \pm 0.2$ & $0.3 \pm 0.1^{*}$ \\
\hline
\end{tabular}

$* P<0.05$ versus sedentary group. Data are expressed as mean \pm SEM. $\mathrm{SD}=$ Standard deviation, RMSSD=Root mean square of the successive differences, LF abs=Low-frequency band absolute, $\mathrm{HF}$ abs $=$ High frequency band absolute, LF=Low-frequency band, HF=High Frequency band, $\mathrm{LF} / \mathrm{HF}=$ Sympathovagal balance, $\mathrm{SEM}=$ Standard error mean, VLF abs $=$ Very LF absolute

predictor of mortality after myocardial infarction, congestive heart failure, and diabetic neuropathy in humans. ${ }^{[2,11]}$ In the present study, we described the use of a ECG platform coupled with ECHO system equipment for measurement of RRI and subsequent analysis of HRV in mice.

Significant advantages of this new approach are: (1) no requirement for surgery, a noninvasive approach and (2) the possibility of combining autonomic evaluation under the same condition as ECO analysis since the determination of heart rate and heart rate variability are based on electrocardiogram (ECG) recordings. Another advantage is the quality of the RRI signal from the ECG, a crucial component for HRV analysis. The ECG platform from the ECO equipment could be used at a sample rate of $8000 \mathrm{~Hz}$, providing a very clear and easily analyzed signal.
Table 2: Echocardiographic evaluations in studied groups

\begin{tabular}{lcc}
\hline & Sedentary & Trained \\
\hline Cardiac structure & \\
ESD $(\mathrm{cm})$ & $0.20 \pm 0.01$ & $0.18 \pm 0.01$ \\
EDD $(\mathrm{cm})$ & $0.34 \pm 0.01$ & $0.33 \pm 0.01$ \\
RWT & $0.48 \pm 0.04$ & $0.69 \pm 0.06 *$ \\
LVM $(\mathrm{mg} / \mathrm{g})$ & $0.0254 \pm 0.0002$ & $0.0274 \pm 0.0004 *$ \\
Systolic function & \\
EF (\%) & $77.5 \pm 2.7$ & $80.3 \pm 4.4$ \\
FS (\%) & $41.7 \pm 1.9$ & $45.8 \pm 3.5$ \\
Diastolic function & $20.0 \pm 0.9$ \\
IVRT (ms) & $1.39 \pm 0.06$ & $14.8 \pm 0.4 *$ \\
E/A & & $1.31 \pm 0.04$ \\
\hline * $P<0.05$ versus sedentary group. Data are expressed as mean \pm SEM. \\
ESD=End-systolic diameter, EDD=End-diastolic diameter, \\
RWT=Relative wall thickness, LVM=Left ventricular mass, EF=Ejection \\
fraction, FS=Shortening fraction, IVRT=Isovolumetric relaxation time, E/ \\
A=E wave/A wave, SEM=Standard error the mean
\end{tabular}

Data obtained in this fashion must be interpreted with caution, as it is well known that restraining and anesthesia can have an influence on physiological parameters. In the preset project, we used an optimum level of ISO anesthesia to ensure the stability of the murine cardiovascular function as well as adequate ventilation and oxygenation for careful match of the artificial ventilation parameters. The literature suggests for proper physiological conditions, that oxygenation, stress levels and myocardial oxygen consumption not deviate from expected physiological ranges and for this reason couple studies concluded an optimum level of $1.5 \%$ of ISO a inhalation anesthetic agent that possess significant advantages over injectable agents such as pentobarbital and ketamine/xylazine. ${ }^{[12,13]}$

In the present study, using the ECG platform of the ECO machine, we recorded RRI and detect similar autonomic changes induced by training, a much simpler method. Thus, 


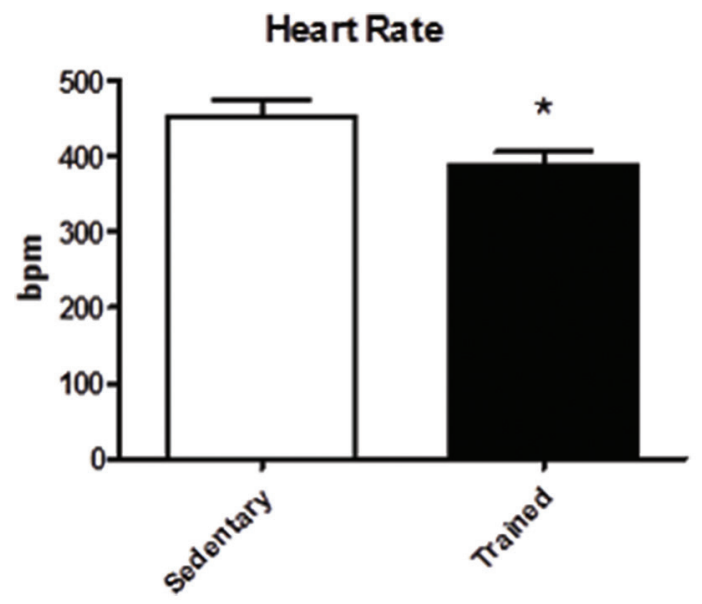

Figure 2: Heart rate in sedentary and trained groups. *Versus Sedentary group

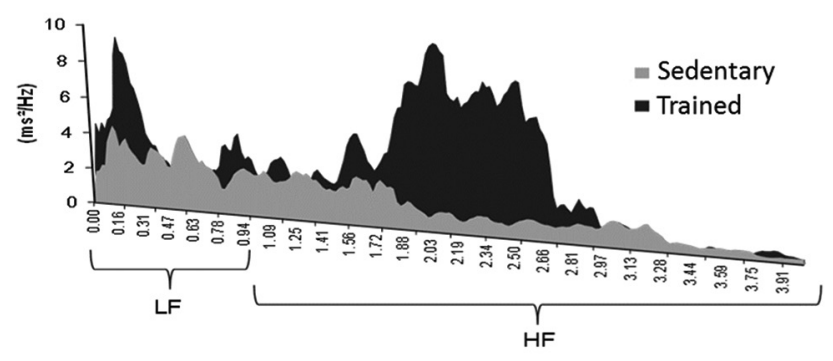

Figure 3: Autonomic power spectrum in mice

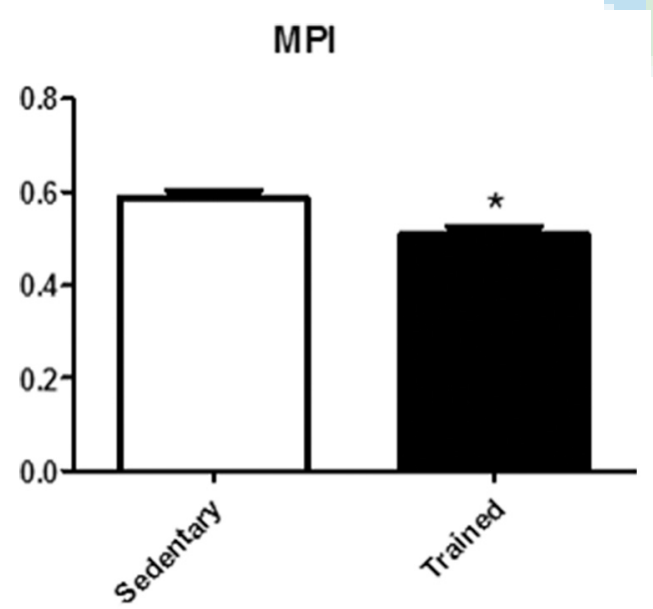

Figure 4: Myocardial performance index in sedentary and trained groups. *Versus Sedentary group

in the present study, the trained group presented an improved autonomic modulation in relation to the sedentary group, showing a reduction of sympathetic modulation and an increase of parasympathetic modulation, which led to a better LF/HF ratio. These evaluations were associated with conventional evaluations provided by ECHO, showing that the trained group had improved cardiac function as compared to the sedentary group.
Aerobic exercise training is often recommended as an important adjuvant therapy for prevention and treatment of cardiac disorders. Indeed, studies have shown that aerobic exercise training improves cardiac function and autonomic modulation in human and animal models..$^{[5,8,14]}$ This can be seen by cardiac adaptations such as an increase in stroke volume, ${ }^{[15,16]}$ adjustments in diastolic and systolic function, ${ }^{[16]}$ and positive changes in cavity diameter and ventricular mass. ${ }^{[17,18]}$ Indeed, we previously observed bradycardia associated with increased cardiac vagal tonus and reduced sympathetic tonus in male mice after 4 weeks of aerobic training on the treadmill. ${ }^{[5]}$ It is important to emphasize that catheters were inserted in carotid artery and jugular vein for blood pressure recording and drugs (atropine and propranolol) injections in mice in this study.

\section{Conclusion}

In conclusion, an ECG platform coupled with an ECHO system enabled the acquisition of the RRI in an appropriate quality for analysis of HRV in mice, allowing associations of morphometric and functional cardiac evaluations with changes in autonomic modulation of the heart in physiological situations.

\section{Authors contributions}

Filipe Fernandes Stoyell-Conti was instrumental to conception and design of the work, acquisition of data, analysis and interpretation of data, statistical analysis and draft of manuscript; he was supported by a fellowship from CAPES. Fernando dos Santos and Jacqueline Freire Machi contributed to acquisition, analysis and interpretation of data; Santos was supported by FAPESP; Diana Rosa Hernandez contributed to conception and design of the work and acquisition of data; Catarina de Andrade Barboza helped with the manuscript preparation; she was supported by a fellowship from FAPESP. Maria Claudia Irigoyen contributed to analysis and interpretation of data; Kátia De Angelis contributed to analysis and interpretation of data and draft of the manuscript; she worked together with Morris to design and implement the project (equal participation). Mariana Morris contributed to conception and design of the work, analysis and interpretation of data, statistical analysis and draft of the manuscript.

\section{Acknowledgments}

CAPES, Nova Southeastern University, Miami VA Healthcare System and Universidade Nove de Julho.

\section{Financial support and sponsorship}

This study was supported by CAPES: 99999.008874/201400 (Dr. Stoyell-Conti), U.S. Department of Defense: W81XWH-13-2-0085 (Dr. Morris) and CNPq-BPQ (Dr. Irigoyen and Dr. De Angelis).

\section{Conflicts of interest}

There are no conflicts of interest.

\section{REFERENCES}

1. Heart rate variability: Standards of measurement, physiological interpretation and clinical use. Task force of the European society 
of cardiology and the North American society of pacing and electrophysiology. Circulation 1996;93:1043-65.

2. Bigger JT Jr., Fleiss JL, Steinman RC, Rolnitzky LM, Kleiger RE, Rottman JN, et al. Frequency domain measures of heart period variability and mortality after myocardial infarction. Circulation 1992;85:164-71.

3. Jaiswal M, Urbina EM, Wadwa RP, Talton JW, D'Agostino RB Jr., Hamman RF, et al. Reduced heart rate variability is associated with increased arterial stiffness in youth with type 1 diabetes: The SEARCH CVD study. Diabetes Care 2013;36:2351-8.

4. Robinson RG, Spalletta G, Jorge RE, Bassi A, Colivicchi F, Ripa A, et al. Decreased heart rate variability is associated with poststroke depression. Am J Geriatr Psychiatry 2008;16:867-73.

5. De Angelis K, Wichi RB, Jesus WR, Moreira ED, Morris M, Krieger EM, et al. Exercise training changes autonomic cardiovascular balance in mice. J Appl Physiol (1985) 2004;96:2174-8.

6. Irigoyen MC, Paulini J, Flores LJ, Flues K, Bertagnolli M, Moreira ED, et al. Exercise training improves baroreflex sensitivity associated with oxidative stress reduction in ovariectomized rats. Hypertension 2005;46:998-1003.

7. Sanches IC, de Oliveira Brito J, Candido GO, da Silva Dias D, Jorge L, Irigoyen MC, De Angelis K. Cardiometabolic benefits of exercise training in an experimental model of metabolic syndrome and menopause. Menopause. 2012 19(5):562-8.

8. Conti FF, Brito Jde O, Bernardes N, Dias Dda S, Malfitano C, Morris M, et al. Positive effect of combined exercise training in a model of metabolic syndrome and menopause: Autonomic, inflammatory, and oxidative stress evaluations. Am J Physiol Regul Integr Comp Physiol 2015;309:R1532-9.

9. Perrino C, Gargiulo G, Pironti G, Franzone A, Scudiero L, De Laurentis M, et al. Cardiovascular effects of treadmill exercise in physiological and pathological preclinical settings. Am J Physiol Heart
Circ Physiol 2011;300:H1983-9.

10. Rodrigues B, Rosa KT, Medeiros A, Schaan BD, Brum PC, De Angelis K, et al. Hyperglycemia can delay left ventricular dysfunction but not autonomic damage after myocardial infarction in rodents. Cardiovasc Diabetol 2011;10:26.

11. Ribeiro JP, Moraes Filho RS. Heart rate variability as an instrument for the investigation of the autonomic nervous system under pathophysiological conditions. Rev Bras Hipertens 2005;12:99-106.

12. Murakami M, Niwa H, Kushikata T, Watanabe H, Hirota $\mathrm{K}$, Ono $\mathrm{K}$, et al. Inhalation anesthesia is preferable for recording rat cardiac function using an electrocardiogram. Biol Pharm Bull 2014;37:834-9.

13. Constantinides C, Mean R, Janssen BJ. Effects of isoflurane anesthesia on the cardiovascular function of the $\mathrm{C} 57 \mathrm{BL} / 6$ mouse. ILAR $\mathrm{J}$ 2011;52:e21-31.

14. Sugawara J, Murakami H, Maeda S, Kuno S, Matsuda M. Change in post-exercise vagal reactivation with exercise training and detraining in young men. Eur J Appl Physiol 2001;85:259-63.

15. Brandao MU, Wajngarten M, Rondon E, Giorgi MC, Hironaka F, Negrao CE, et al. Left ventricular function during dynamic exercise in untrained and moderately trained subjects. J Appl Physiol (1985) 1993;75:1989-95.

16. D'Andrea A, Caso P, Severino S, Galderisi M, Sarubbi B, Limongelli G, et al. Effects of different training protocols on left ventricular myocardial function in competitive athletes: A Doppler tissue imaging study. Ital Heart J 2002;3:34-40.

17. Shapiro LM, Smith RG. Effect of training on left ventricular structure and function. An echocardiographic study. Br Heart J 1983;50:534-9.

18. Dart AM, Meredith IT, Jennings GL. Effects of 4 weeks endurance training on cardiac left ventricular structure and function. Clin Exp Pharmacol Physiol 1992;19:777-83.

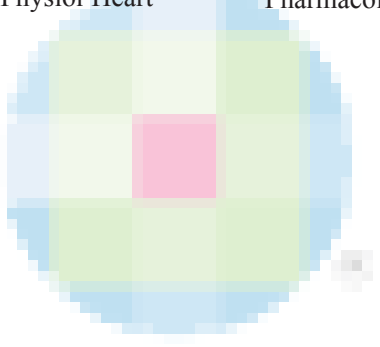

